\title{
BMJ Open Infant TB Infection Prevention Study (iTIPS): a randomised trial protocol evaluating isoniazid to prevent $M$. tuberculosis infection in HIV-exposed uninfected children
}

\author{
Sylvia M LaCourse (1) , ${ }^{1}$ Barbra A Richardson, ${ }^{2,3}$ John Kinuthia, ${ }^{4,5}$ A J Warr, ${ }^{6,7}$ \\ Elizabeth Maleche-Obimbo, ${ }^{8}$ Daniel Matemo, ${ }^{4}$ Lisa M Cranmer, ${ }^{9,10}$ \\ Jaclyn N Escudero, ${ }^{3}$ Thomas R Hawn, ${ }^{1}$ Grace C John-Stewart (D) 1,3,11,12
}

To cite: LaCourse SM, Richardson BA, Kinuthia J, et al. Infant TB Infection Prevention Study (iTIPS): a randomised trial protocol evaluating isoniazid to prevent $M$. tuberculosis infection in HIV-exposed uninfected children. BMJ Open 2020;10:e034308. doi:10.1136/ bmjopen-2019-034308

- Prepublication history and additional material for this paper are available online. To view these files, please visit the journal online (http://dx.doi org/10.1136/bmjopen-2019034308).

Received 13 September 2019 Revised 05 December 2019 Accepted 07 January 2020

Check for updates

(C) Author(s) (or their employer(s)) 2020. Re-use permitted under CC BY-NC. No commercial re-use. See rights and permissions. Published by BMJ.

For numbered affiliations see end of article.

Correspondence to Dr Sylvia M LaCourse; sylvial2@uw.edu

\section{ABSTRACT}

Introduction HIV-exposed uninfected (HEU) infants in tuberculosis (TB) endemic settings are at high risk of Mycobacterium tuberculosis (Mtb) infection and TB disease, even in the absence of known Mtb exposure. Because infancy is a time of rapid progression from primary infection to active TB disease, it is important to define when and how TB preventive interventions exert their effect in order to develop effective prevention strategies in this high-risk population.

Methods and analysis We designed a non-blinded randomised controlled trial to determine efficacy of isoniazid (INH) to prevent primary Mtb infection among HEU children. Target sample size is 300 (150 infants in each arm). Children are enrolled at 6 weeks of age from maternal and child health clinics in Kenya and are randomised to receive 12 months of daily $\mathrm{INH} \sim 10 \mathrm{mg} /$ $\mathrm{kg}$ plus pyridoxine or no INH. The primary endpoint is Mtb infection, assessed by interferon-gamma release assay QuantiFERON-TB Gold Plus (QFT-Plus) or tuberculin skin test after 12 months post-enrolment. Secondary outcomes include severe adverse events, expanded Mtb infection definition using additional QFT-Plus supernatant markers and determining correlates of Mtb infection. Exploratory analyses include a combined outcome of TB infection, disease and mortality, and sensitivity analyses excluding infants with baseline TB-specific responses on flow cytometry.

Ethics and dissemination An external and independent Data and Safety Monitoring Board monitors adverse events. Results will be disseminated through peerreviewed journals, presentations at local and international conferences to national and global policy-makers, the local community and participants.

Trial registration number NCT02613169; Pre-results.

\section{INTRODUCTION}

HIV-exposed uninfected (HEU) infants in tuberculosis (TB) endemic settings have a high risk of Mycobacterium tuberculosis (Mtb) infection and TB disease, even in absence of
Strengths and limitations of this study

- Most children born to mothers living with HIV are HIV-exposed and uninfected (HEU) but remain at high risk of tuberculosis (TB), making them an important population in which to study TB prevention.

- Current TB prevention guidelines do not recommend routine isoniazid (INH) preventive therapy (IPT) for children (including HEU) without a known TB contact, though recent data suggest the majority of transmission to children occurs outside the household due to community or unperceived household TB; therefore, a strength of our study is the enrolment of children without known TB exposure.

- Because of the high risk of progression to TB disease in infants, our strategy, which focuses on prevention of primary Mycobacterium tuberculosis (Mtb) infection as detected by a combined endpoint of interferon-gamma release assays and tuberculin skin test is novel, despite known limitations of both assays.

- Widespread implementation of IPT in adult people living with HIV (including peripartum women) could significantly decrease TB risk in infants, making an HEU-focused TB prevention strategy less needed.

- Given equipoise regarding whether INH prevents Mtb infection in general, and a lack of data specifically among HEU children, a randomised controlled trial design would provide important information regarding INH efficacy for primary prevention in this high-risk population.

known Mtb exposure. ${ }^{1-3}$ Because infancy is a time of rapid progression from primary infection to active $\mathrm{TB},{ }^{4}$ it is important to know how TB preventive interventions exert their effects to build new strategies that adapt or extend approaches used in adults. Protecting HEU infants during this vulnerable period of immunodeficiency may provide long-term benefits. 


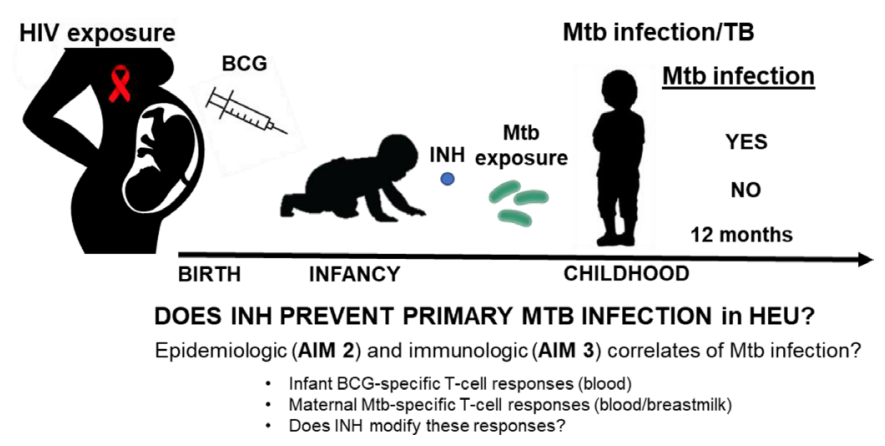

Figure 1 Study schema: aims of a randomised controlled trial to evaluate isoniazid (INH) to prevent Mycobacterium tuberculosis (Mtb) infection in HIV-exposed uninfected (HEU) infants.

Children have a higher rate of progression from Mtb infection to active TB disease than adults. ${ }^{56}$ In young children who lack pre-existing adaptive immune responses, Mtb infection may progress rapidly to $\mathrm{TB}$ disease, and both innate and early adaptive immune responses likely influence susceptibility. ${ }^{5-9}$ Virtually all childhood TB disease reflects primary disease, in contrast to adults where a significant portion of disease is due to reactivation of latent TB infection. ${ }^{10}$ World Health Organization (WHO) recommends TB preventive therapy, including isoniazid (INH) preventive therapy (IPT), be provided to people living with HIV (PLHIV) $>12$ months of age to prevent TB. ${ }^{11}$ Among children living with HIV (CLHIV), three randomised controlled trials (RCTs) yielded conflicting data regarding whether INH prevents TB disease and/ or mortality. ${ }^{212-14}$ Only one evaluated HEU infants and found no protective effect in decreasing a composite outcome of TB disease, Mtb infection (as measured by tuberculin skin test (TST)) or mortality. ${ }^{2}$ While previous RCTs have focused on prevention of TB disease, there are scant data regarding INH impact on primary Mtb

\begin{tabular}{|l|l|}
\hline Study Design & Non-blinded randomised controlled trial \\
\hline Intervention & $\begin{array}{l}\text { Intervention: INH for 12 months } \\
\text { Control group: No INH }\end{array}$ \\
\hline $\begin{array}{l}\text { Primary } \\
\text { Outcomes }\end{array}$ & $\begin{array}{l}\text { Aim 1: Mtb infection in HEU infants at 12 months post- } \\
\text { enrolment as measured by IGRA (QFT-Plus) and/or TST } \\
\text { Aim 2: Epidemiologic correlates of infant Mtb infection } \\
\text { Aim 3: Immunologic correlates of infant Mtb infection }\end{array}$ \\
\hline Population & $\begin{array}{l}\text { HEU infants 6 weeks of age and their HIV-infected } \\
\text { mothers }\end{array}$ \\
\hline Exclusions & $\begin{array}{l}\text { - Infants with known exposure to active TB in household } \\
\text { - Positive HIV DNA at 6 weeks } \\
\text { - Premature and/or birthweight <2.5 kg }\end{array}$ \\
\hline $\begin{array}{l}\text { Target } \\
\text { Enrolment }\end{array}$ & $\begin{array}{l}\text { 300 HEU infants and their HIV-infected mothers } \\
\text { (150 each arm) }\end{array}$ \\
\hline $\begin{array}{l}\text { Sampling } \\
\text { Framework }\end{array}$ & $\begin{array}{l}\text { Consecutive enrolment of HEU infants and their HIV- } \\
\text { infected mothers at MCH/PMTCT clinics in western } \\
\text { Kenya }\end{array}$ \\
\hline
\end{tabular}

Figure 2 Overall study strategy. HEU, HIV-exposed uninfected; IGRA, interferon-gamma release assay; INH, isoniazid; $\mathrm{MCH}$, maternal child health; Mtb, Mycobacterium tuberculosis; PMTCT, prevention of maternal to child transmission; TST, tuberculin skin test. infection. Among 6-month-old HEU infants in Kenya, we found $10 \%$ had evidence of Mtb infection by interferongamma release assays (IGRAs), suggesting a potential $20 \%$ annual cumulative incidence of Mtb infection. ${ }^{15}$ In a South African birth cohort including 22\% HIV-infected mothers, TST test conversion incidence was 11.8 per 100 child-years, with the majority of conversions occurring before 1 year of age. ${ }^{16}$ Among Ugandan children (median age 36 months), prevalence of Mtb infection (either TST or positive IGRA) was two-fold higher among HEU compared with HIV-unexposed children (HUU), with higher prevalence of TST positivity versus IGRA in both groups (HEU TST $27.2 \%$ vs IGRA $6.4 \%$, HUU TST $20.6 \%$ vs $1.5 \%) .{ }^{17}$ This suggests HEU infants have a substantial incidence of Mtb infection, ${ }^{118}$ as well as low to modest concordance between different Mtb infection measures.

There is currently no gold standard for Mtb infection diagnosis. ${ }^{19}$ While TST is recommended in children under 5 years of age,${ }^{2021}$ false positivity due to BCG vaccination can occur. ${ }^{22}$ Data regarding IGRA performance in young children are limited; however, a recent study of BCG-immunised infants in South Africa indicated high QuantiFERON interferon gamma (IFN- $\gamma$ ) conversion values were strongly associated with subsequent development of TB. ${ }^{23}$ Because IGRAs offer increased specificity in the presence of recent BCG vaccine, it is plausible they could enhance the ability to measure the preventive effect of INH. ${ }^{22}$ Cross-reactivity to non-TB mycobacteria may cause false positives in both IGRA and TST. ${ }^{19}$ IGRA and TST agreement in children varies widely and appears affected by nutrition and HIV status, TB burden and BCG immunisation. ${ }^{24-28}$ Recent American Thoracic Society (ATS) /Infectious Disease Society of America/Centers for Disease Control and Prevention guidelines recommend dual testing with IGRA and TST for groups who are both likely to be infected and at high risk of progression to TB disease as a strategy to increase diagnostic sensitivity. ${ }^{21}$ Reduction of specificity with this strategy may be acceptable when consequences of missed Mtb infection (and therefore missed opportunity for treatment) outweigh risks of therapy-associated adverse events. Few longitudinal studies among young infants including HEUs with serial IGRA and TST testing exist. ${ }^{23} 29$ A prospective infant HEU cohort using both IGRA and TST can provide an efficient approach to probe determinants of Mtb infection, more rapidly accruing endpoints (Mtb infection) than studies of TB disease. This study design can contribute unique insights regarding prevention strategies.

Kenyan guidelines mirror WHO and recommend IPT for all known TB-exposed children $<5$ years of age and all CLHIV $>1$ year of age regardless of TB exposure. ${ }^{1130}$ However, for children $<5$ years without known TB exposure (including HEU), and for CLHIV $<1$ year, IPT is not recommended. ${ }^{11}{ }^{30}$ These guidelines illustrate uncertainty regarding IPT in young children, following an RCT from South Africa/Botswana that failed to demonstrate IPT effectiveness in preventing TB disease among CLHIV and HEU <1 year of age without known TB 
exposure. ${ }^{212-14}$ It remains possible that among HEU children exposed to unperceived community or household TB, INH may prevent primary Mtb infection. Although data are conflicting, some adult studies have demonstrated IPT benefit in TST-negative or IGRA-negative adult PLHIV suggesting IPT may confer protection from Mtb infection. ${ }^{31-34}$

The primary goal of this study is to determine whether INH prevents primary Mtb infection in HEU infants, to determine timing and cofactors of primary Mtb acquisition in the first year of life, and to examine the role of immune protective mechanisms in this cohort (figure 1). This paper details the study protocol of an RCT evaluating efficacy of a 12-month course of INH to prevent Mtb infection as measured by IGRA and/or TST in HEU children enrolled at 6 weeks of age in western Kenya.

\section{METHODS AND ANALYSIS}

\section{Study design}

The infant TB Infection Prevention Study ('iTIPS') is a two-arm, non-blinded RCT comparing efficacy of a 12-month course of daily INH versus no INH to prevent Mtb infection among HEU Kenyan children enrolled at
6 weeks of age (figure 2). Eligible infants are randomised using a 1:1 allocation to INH versus no INH (figure 3).

\section{Study sites}

Kenya is one of 22 high TB burden countries with a generalised TB epidemic, ${ }^{35}$ with an estimated TB prevalence of 426 per $100000 .^{36}$ This study is conducted in collaborative research sites in western Kenya embedded in Ministry of Health (MOH) maternal child health $(\mathrm{MCH})$ clinics. HIV-infected mothers are followed as part of the national prevention of maternal to child transmission (PMTCT) programme and currently receive Option B+ triple antiretroviral therapy (ART). ${ }^{37}$ Per Kenyan guidelines, all PLHIV should be screened at routine HIV care visits using symptom-based TB screening and those with negative screens are evaluated for IPT. ${ }^{113537}$

\section{Recruitment processes and eligibility criteria}

We recruit mothers living with HIV and their HIVexposed infants from MCH/PMTCT sites. Infants 6 weeks (+4 weeks) of age are eligible for inclusion if they are born to HIV-infected mothers, with birth weight $\geq 2.5 \mathrm{~kg}$, and not born premature ( $\geq 37$ weeks' gestation). Infants

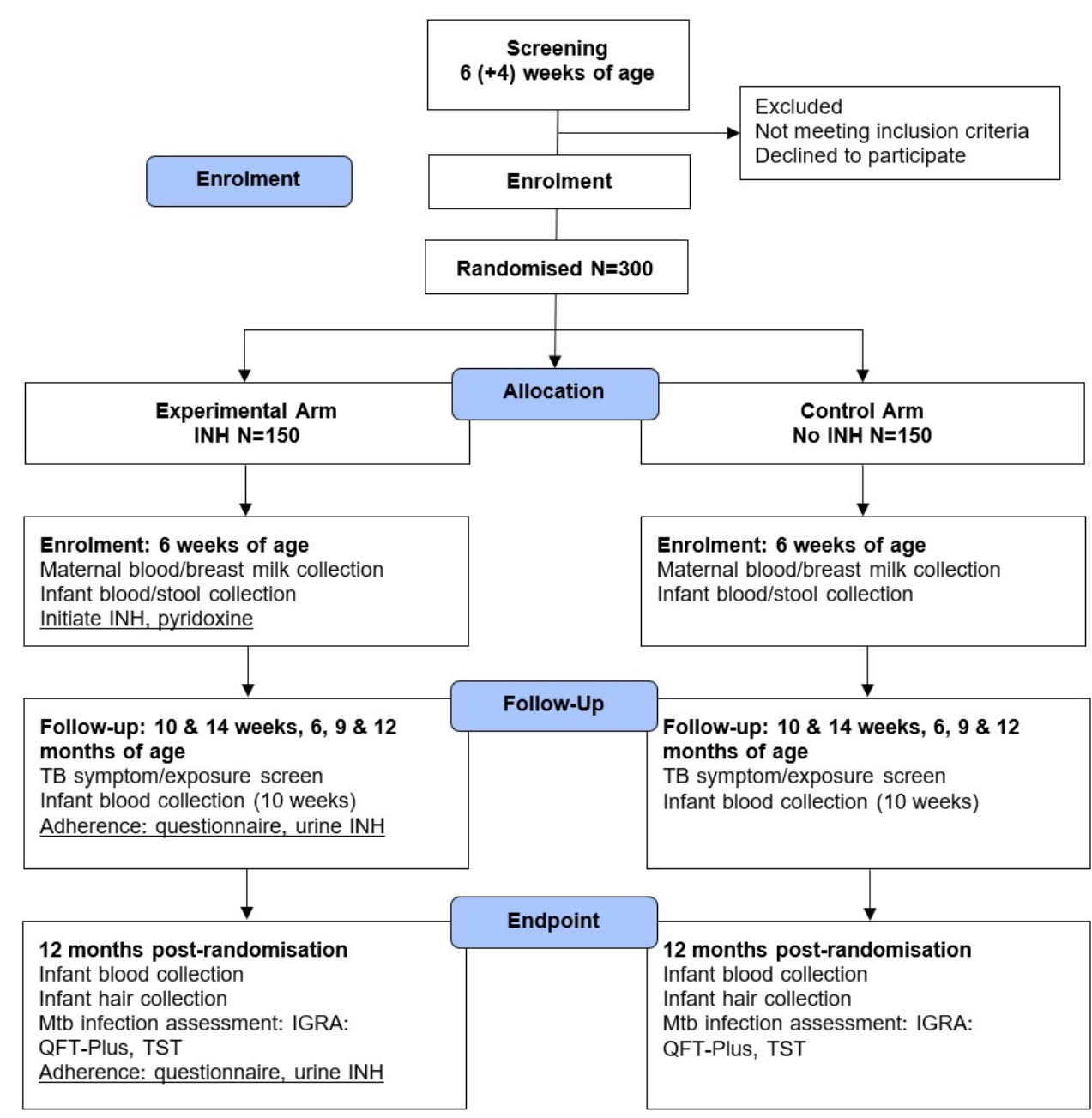

Figure 3 CONSORT diagram. IGRA, interferon-gamma release assay; INH, isoniazid; Mtb, Mycobacterium tuberculosis; TST, tuberculin skin test. 
with known household TB exposure, including mothers with TB diagnosed in the past year, are ineligible. Infants enrolled in other TB prevention or TB vaccine studies are ineligible because these interventions might affect ascertainment of endpoints.

\section{Randomisation}

Site-stratified randomisation is used to allocate infants 1:1 to INH or no INH arms. Randomisation numbers were generated prior to study start using STATA V.14 "ralloc" command with resulting randomisation assignment by participant ID printed on cards and placed in opaque envelopes.

\section{Blinding}

The study is non-blinded to enable prompt clinical management for any potential drug-related adverse event. IGRAs are performed in the Kenya Medical Research Institute (KEMRI) Centers for Disease Control (CDC) laboratory, which is blinded to arm. The study team administers TST and is not blinded to TST result. Data monitoring by the study team is not disaggregated by study arm. The study biostatistician reviews data by arm during preparation of reports to the external Data and Safety Monitoring Board (DSMB). These data are reviewed during closed DSMB sessions, which excludes team members involved in study implementation.

\section{Enrolment and study procedures \\ Enrolment}

After informed consent is obtained by study staff, household locator information, medical identification number and cellphone contacts are obtained to facilitate tracing. On enrolment, standardised questionnaires regarding sociodemographic, clinical, obstetric and HIV-related factors, TB exposure and history, and TB symptoms (for infant, mother and household members (by maternal report) using WHO symptom screen ${ }^{38}$ ) are administered (online supplementary table 1). Mothers with suspected $\mathrm{TB}$ are referred to the TB programme for further screening. If mothers are found to have TB on enrolment, their infants are ineligible for participation and are referred to receive INH per Kenya national guidelines.

Infants undergo physical examination measuring weight, height/length, mid-upper arm circumference and presence of BCG scar. Medical records are used to abstract data on infant birth weight, PMTCT prophylaxis, other medications, immunisations, and maternal ART regimen, viral load and CD4 cell counts.

\section{Intervention}

INH $\sim 10 \mathrm{mg} / \mathrm{kg}(7-15 \mathrm{mg} / \mathrm{kg})$ is administered once daily to infants in the INH arm for 12 months. Standardised weight-based dosing (by weight band using $100 \mathrm{mg}$ scored tablets) is used, corresponding to Kenya and WHO recommendations. ${ }^{3039}$ Pyridoxine is provided to children randomised to INH to decrease peripheral neuropathy risk. ${ }^{30}{ }^{39}$ Caregivers are advised on how to pulverise INH and pyridoxine to be mixed with small quantities of breast milk, clean water or liquid co-trimoxazole to ensure full doses are given and for ease of administration to infants. Participants in the intervention arm are administered daily INH and pyridoxine by caregivers. Infants in the control arm do not receive INH or pyridoxine.

\section{Participant follow-up}

Follow-up visits occur at 10 weeks for infants enrolled at 6 weeks of age and at 14 weeks, and 6, 9 and 12 months of age for all participants coinciding with routine Kenya paediatric visit schedule. Follow-up visits include assessment of any TB diagnosis in the mother, infant or household member since the past visit, as well as any TB symptom in the mother, infant and household members (by maternal report). Infants in the no INH arm found to have a known TB contact during the study are referred for IPT per Kenyan guidelines. Infants and mothers found to have TB symptoms are referred to the $\mathrm{MOH}$ TB Programme for further evaluation. Questionnaires regarding caregiver barriers and facilitators to providing prophylactic medications (co-trimoxazole, antiretrovirals for PMTCT and INH (if in INH arm)) are administered at the 6 month of age visit. Endpoint ascertainment occurs at a study visit 12 months post-randomisation at approximately 14 months of age.

\section{Sample collection}

Infant blood for peripheral blood mononuclear cells (PBMCs) and plasma are collected at baseline and visit 2 (10-14 weeks of age). At 12 months post-randomisation, blood is collected for IGRA (QFT-Plus) and TST placed and read within $48-96$ hours. ${ }^{4041}$ Infant rectal swabs are collected at enrolment for future gut microbiome studies. Maternal breast milk and blood for PBMC and plasma separation are collected on enrolment.

\section{Study procedures specific to infants randomised to INH}

Liver function tests (LFTs) are performed at enrolment and 1 month following INH initiation. Adherence is assessed by caregiver report at follow-up visits. Urine is collected at follow-up and study endpoint visits and tested using strips developed to detect INH metabolites. ${ }^{42} 43$ Hair is collected at study endpoint for future assessment of INH levels as a more objective adherence measure over time. $^{445}$

\section{Safety considerations}

IPT has been shown to be safe in prior RCTs and is administered routinely to TB-exposed infants. ${ }^{2}{ }^{12-14}$ Although routine LFT monitoring is not recommended during INH in children, ${ }^{20}$ for this trial baseline LFTs are drawn at enrolment and 1 month after INH initiation. National Institutes of Health (NIH) Division of AIDS (DAIDS) Table for Grading the Severity of Paediatric Adverse Events is used to grade toxicities. ${ }^{46}$ Infants with LFTs $\leq$ grade 2 are allowed to initiate INH. Infants with baseline LFTs $\geq$ grade 3 at baseline have LFTs monitored every 2 weeks and do not initiate INH until LFTs are $\leq$ grade 2. Children are evaluated for peripheral neuropathy using a 
truncated Denver Developmental test. ${ }^{47}$ After INH initiation, if toxicity is suspected, study-administered drugs are immediately discontinued and in case of concern for hepatoxicity, LFTs are repeated.

An external and independent DSMB, including experts in paediatric TB, biostatistics and trial design, monitors severe adverse events (SAEs). Summaries of SAEs are given to DSMB members during scheduled meetings. Each SAE is assigned plausibility of relatedness to study drug by study investigators. 'Open' reports detailing cumulative overall SAEs are descriptive (no statistical analyses). 'Closed' reports of SAEs by study arm are reviewed and the DSMB makes recommendations regarding any imbalances in safety outcomes. O'Brien-Fleming boundaries for benefit and harm are used for interim monitoring, and these boundaries are provided by the study statistician in closed reports. The DSMB assesses operational aspects, safety and effectiveness, and makes recommendations regarding study continuation or modifications. Futility is not considered a basis for stopping rules because of the trial's value in understanding correlates of Mtb infection in HEU infants.

\section{Discontinuation, withdrawal or allocation modification}

Participants may withdraw from the study at any point. Study investigators may withdraw a participant on a caseby-case basis if the intervention or study involvement poses a risk to the participant. No modification of allocation will be made. Infants who receive at least one dose of study drug will be included in per-protocol analyses. Caregivers of infants who discontinue INH are encouraged to continue study follow-up and endpoint ascertainment.

\section{Data collection and management}

Study staff use tablets to collect de-identified data using secure password-protected Research Electronic Data Capture mobile software (REDCap; Vanderbilt University, Nashville, Tennessee, USA). ${ }^{48}$ Data are uploaded daily from tablets to the web-based REDCap database. Study investigators will have access to the finalised dataset.

\section{Patient and public involvement}

Patients were not directly involved in the development of the research question, design of the study or recruitment. We assess the burden of the trial intervention for participants by gathering data on adverse events, tolerability of INH, and assessment of caregiver barriers and facilitators to providing prophylactic medications to HEU children via questionnaire. Overall study results will be shared with the clinical facilities and presented to local stakeholders including $\mathrm{MOH}$ county and national representatives.

\section{Outcome measures}

The primary outcome is Mtb infection by QFT-Plus assay or TST 12 months after enrolment. Similar to QuantiFERON-TB Gold (QFT) IGRA, QFT-Plus measures IFN $-\gamma$ released by primarily CD4+ T helper lymphocytes after TB-specific antigen (ESAT-6 and CFP-10) stimulation. In addition, QFT-Plus measures IFN- $\gamma$ released by
CD8+ cytotoxic $\mathrm{T}$ lymphocytes, after stimulation with the same antigens, which may have increased sensitivity in children, and in populations with lower CD4 counts including PLHIV ${ }^{49}{ }^{50}$ Responses of $\geq 0.35 \mathrm{IU} / \mathrm{mL}$ to TB antigens above the Nil response in either the primarily $\mathrm{CD} 4+$ (TB1) or CD8+ response (TB2) (with $\mathrm{Nil}<8 \mathrm{IU} / \mathrm{mL}$ and a positive mitogen control) are considered positive per manufacturer recommendations ${ }^{49}$ A TST of $\geq 10 \mathrm{~mm}$ is considered positive. ${ }^{20}$

Secondary outcomes include severe adverse events (grade $\geq 3$ per DAIDS Grading Severity of Paediatric Adverse Experiences), ${ }^{46}$ use of IFN- $\gamma$-independent immune markers in QFT-Plus supernatants to indicate Mtb infection, ${ }^{51-55}$ and epidemiological and immunological correlates of Mtb infection. Exploratory outcomes include combined endpoint of Mtb infection, TB diagnosis and/or death, as well as sensitivity analyses of the primary outcome of Mtb infection after excluding infants with evidence of immune responses to ESAT- 6 or CFP-10 at enrolment in flow cytometric analyses.

\section{Sample size and power analysis}

Assuming an alpha of 0.05 , power of 0.80 , using a twosided test and a 1:1 allocation ratio, with 125 infants in each arm, we have power to detect at least a $65 \%$ decrease in Mtb infection in INH arm versus control if cumulative incidence of Mtb infection in the control arm at 12 months is 0.20 , or to detect $70 \%-80 \%$ or higher (HR $0.3-0.2$ ) decrease if cumulative incidence of Mtb infection in the control arm is 0.15 or 0.10 (online supplementary table 2). To account for loss to follow-up, non-adherence and INH resistance, we increased sample size by $20 \%$, with goal enrolment of 300 infants (150 per arm). Baseline characteristics will be compared between randomisation arms to assess randomisation adequacy.

\section{Statistical methods and analysis}

\section{Primary outcome}

Modified intention-to-treat: We will use a modified intentionto-treat approach, including all participants who underwent randomisation irrespective of receiving trial medication with at least one measure of Mtb infection (QFT-Plus or TST), excluding children found to be HIV DNA positive during the study. We will compare the proportion of infants with Mtb infection (either QFT or TST positive) at 12 months between INH and no INH arms using a $\chi^{2}$ test and estimate relative risk with $95 \%$ CIs using a generalised linear model with log link and Poisson family. We will compare cumulative incidence of Mtb infection by arm using a Cox proportional hazard regressions model.

Per protocol: We will evaluate our primary outcome by a per-protocol analysis, considering only HEU infants who took at least one dose as taking INH versus infants who did not take any INH. We anticipate future sensitivity analyses using IPT adherence and continuation data as exposure of interest and Mtb infection as outcome. 


\section{Secondary outcome}

Safety and expanded Mtb infection outcomes: For secondary outcomes, we will compare proportions of participants by arm using either $\chi^{2}$ or Fisher's exact tests as appropriate for $\geq$ grade 3 serious adverse events. In addition, we will conduct secondary analyses using an expanded Mtb infection definition including a positive TST, QFTPlus or IFN- $\gamma$-independent immune markers in QFT-Plus supernatants.

Epidemiological and immune correlates of Mtb infection will be assessed using nested case-control studies incorporating all Mtb infections from both arms then conducting stratified analyses in each trial arm to evaluate potential cofactors modified by INH.

\section{Exploratory outcome}

We will compare a composite endpoint of Mtb infection, TB diagnosis and/or death between randomisation groups using a $\chi^{2}$ test. Baseline assays may detect evidence of Mtb infection. We will conduct additional exploratory analyses, incorporating data from baseline assays ${ }^{56}$ (using flow cytometry of cryopreserved PBMCs) to exclude infants with evidence of Mtb-specific immune responses to ESAT-6 or CFP-10 at enrolment also using a $\chi^{2}$ test.

\section{ETHICS AND DISSEMINATION}

Informed consent is obtained from caregivers. Any protocol changes will be approved by relevant ethical review boards. The protocol is available online (http:// depts.washington.edu/gwach/wp-content/uploads / 2012/10/iTIPS_Protocol_v1.8_01Nov2019.pdf).

We will share trial results at study sites, and with regional and national policy-makers. We plan on submitting final results as a peer-reviewed manuscript and will use International Committee of Medical Journal Editors authorship criteria. Study investigators will collaborate in writing final study results.

HEU children are at increased risk for Mtb infection and TB disease. IPT is not routinely provided to HEU infants in Kenya without evidence of exposure to a known TB case. There is mixed evidence regarding IPT effectiveness to prevent TB disease in infants $<1$ year. Given potential benefits of IPT to prevent Mtb infection, heightened risk for Mtb infection in this population and safety of intervention, there is equipoise for randomisation.

\section{Trial status}

Trial recruitment and enrolment began 15 August 2016. Participant follow-up is anticipated to complete October 2019, with laboratory analyses of the QFT-Plus endpoint anticipated to be completed in December 2019.

\section{DISCUSSION}

INH has proven benefit to treat latent TB infection and prevent active TB disease in HIV-infected and HIVuninfected populations. ${ }^{57-61}$ Data from adult studies in Botswana, South Africa and Ivory Coast indirectly suggest IPT may prevent Mtb infection; TST-negative adult PLHIV who received IPT were protected from active TB, suggesting IPT may both prevent Mtb infection and progression to TB disease. ${ }^{31-34}$ IPT has had variable protective efficacy to prevent TB disease and mortality in CLHIV. $^{2-14}$ An RCT in South Africa in the pre-ART era randomised CLHIV $\geq 8$ weeks of age to INH versus placebo independent of reported TB exposure and found INH prevented TB disease by $70 \%$ and decreased mortality by $54 \%$, leading to early trial discontinuation. ${ }^{12}$ In the observational extension of the trial, combination IPT and ART further decreased TB risk by $11 \% .{ }^{62}$ However, in a pilot study of CLHIV on ART (median age 35 months) not powered for efficacy, IPT did not exert a significant protective effect on active TB (1.5 vs 2.9 TB cases per 100 person-years, incidence rate ratio 0.51 (95\% CI 0.15 to $1.75)) .{ }^{13}$ Similarly, an RCT of INH given for 96 weeks in HIV-infected and HEU infants enrolled at 91-120 days of life in South Africa and Botswana without reported TB exposure did not prevent TB disease in either group. ${ }^{2}$ Furthermore, among HEU, INH did not prevent Mtb infection as measured by a single TST at week 96. In summary, IPT is effective in adults and variably effective for preventing TB disease in HIV-infected and HEU infants, and no trial to date has been designed specifically to evaluate efficacy of IPT to prevent Mtb infection in either adults or children, including both IGRA and TST as an endpoint to maximise sensitivity to identify Mtb infection.

\section{Study limitations}

Enrolment sites are limited to two counties in western Kenya and may not be generalisable to other settings. This area was chosen due to high HIV/TB burden, as well as longstanding collaborations with study investigators in enrolling women and children from MCH/PMTCT clinics. With non-blinded trials, there are concerns about differential reporting and clinical management. However, one of the composite endpoint components (IGRA) is assessed in the KEMRI CDC laboratory, which is blinded to participant INH status. This endpoint is robust and not influenced by unblinded trial design. We have estimated a substantive INH effect ( $65 \%$ decrease), consistent with TB prevention literature for reduction of TB disease among TST-positive adult PLHIV ${ }^{60}$ but undefined for Mtb infection risk. A larger sample size may be useful if Mtb infection prevalence is lower than anticipated or if INH is less effective in prevention of Mtb infection. We have extended post-trial observational follow-up to 24 months of age to assess longer-term Mtb infection incidence. This extended follow-up will allow us to better understand timing of Mtb infection acquisition; however, results will not be included in the primary trial results because the extended observational period will not include receipt of IPT.

There remains a lack of a gold standard to diagnose Mtb infection $^{19}$; both TST and IGRA are indirect measures of 
Mtb infection requiring both infection with Mtb and a functioning immune system to mount a positive response. We have incorporated both tests within our composite primary outcome. TST at 12 months may be positive due to BCG exposure at birth rather than Mtb infection. Age at immunisation and TST testing timing after BCG administration appears to affect TST reactivity, with younger age at BCG immunisation associated with shorter duration of TST reactivity than in adults. In a meta-analysis of 24 studies with $>240000$ participants, among those who were BCG-vaccinated as infants, $<1 \%$ were TST positive after 10 years post-BCG administration, compared with $21 \%$ of participants vaccinated after their first birthday who remained TST positive after 10 years post-BCG. ${ }^{63}$ Similarly, in a recent long-term follow-up study of a BCG versus placebo trial among Native Americans/Alaskan Natives, BCG administered after 1 year of age was associated with increased incidence of TST reactivity extending up to 55 years after vaccination. ${ }^{64}$ Importantly, there are scant data on TST reactivity among BCG-immunised infants TST tested during the first year of life. Among 250 Navajo infants immunised with BCG as newborns, $31 \%$ had TST $\geq 10 \mathrm{~mm}$ at 3 months which reduced to zero at 9 months of age, suggesting rapid waning of BCG-associated TST responses in children receiving BCG at birth. ${ }^{65}$ Therefore, it appears that TST testing at approximately 1 year of age among children immunised with BCG at birth is more likely to represent Mtb infection, as opposed to BCG-induced reactivity. Non-tuberculosis mycobacteria (NTM) can lead to false positives for both IGRA and TST. ${ }^{19}$ Prevalence of NTM disease in Kenya is unknown, but in a recent study evaluating 2900 infants for TB incidence in Kenya, 2.6\% of infants evaluated for TB had NTMs isolated, though none met ATS criteria for NTM disease ${ }^{66}$ Importantly, the study evaluated detection of mycobacteria rather than detection of subclinical NTM infection, and there are currently no standard measures for NTM infection. Our study does not include qualitative work to investigate issues of adherence, though it does include closed-ended questions regarding caregiver barriers and facilitators to providing prophylactic medications to HEU children.

Kenya endorsed routine IPT for PLHIV in 2014 national guidelines, ${ }^{37}$ and counties in which this study is located have had a rapid expansion of IPT as part of routine HIV care. We have described high IPT use in peripartum women. ${ }^{67}$ Widespread IPT implementation in adult PLHIV could significantly decrease TB risk in infants, making an HEU-focused TB prevention strategy less needed. Maternal IPT use is not an exclusion criterion. Infant INH drug exposure through breast milk is very low ${ }^{68}$ and unlikely to exert a direct protective effect in the control arm.

Given equipoise regarding whether INH prevents Mtb infection in general, and a lack of data specifically among HEU children, an RCT design could provide important information regarding INH efficacy for primary prevention in this high-risk population.

\section{Author affiliations}

${ }^{1}$ Department of Medicine, Division of Allergy and Infectious Diseases, University of Washington, Seattle, Washington, USA

${ }^{2}$ Department of Biostatistics, University of Washington, Seattle, Washington, USA

${ }^{3}$ Department of Global Health, University of Washington, Seattle, Washington, USA

${ }^{4}$ Research and Programs, Kenyatta National Hospital, Nairobi, Kenya

${ }^{5}$ Department of Reproductive Health, Kenyatta National Hospital, Nairobi, Kenya

${ }^{6}$ Department of Pediatrics, Baylor College of Medicine, Houston, Texas, USA

${ }^{7}$ Department of Medicine, Baylor College of Medicine, Houston, Texas, USA

${ }^{8}$ Department of Pediatrics and Child Health, University of Nairobi, Nairobi, Kenya

${ }^{9}$ Department of Pediatrics, Division of Infectious Diseases, Emory University, Atlanta, Georgia, USA

${ }^{10}$ Children's Healthcare of Atlanta Inc, Atlanta, Georgia, USA

${ }^{11}$ Department of Epidemiology, University of Washington, Seattle, Washington, USA

${ }^{12}$ Department of Pediatrics, University of Washington, Seattle, Washington, USA

Acknowledgements We would like to acknowledge the iTIPS study staff (Jepharson Mecha, Celestine Adogo, Jovine Akinyi Mbata, Kevin Sellanga Ochieng, Mercy Olilo, Aggrey Olimba, Cynthia Achieng Ongete, Esther Achieng, Dancun Otieno Aroko, Elizabeth Wanjiku Mwangi), the Kisumu and Siaya County Directors of Health, health facility staff, UW-Kenya and Kenyatta National Hospital Research and Programs operational staff (including Emma Nzove). Qiagen provided discounted QFT-Plus test kits, and the Kenya Medical Research Institute (KEMRI) CDC in Kisumu, Kenya performs the QFT-Plus assays. We thank the University of Washington Global Center for the Integrated Health of Women, Adolescents, and Children (Global WACh) for comments and insights provided during study design and manuscript development. Most of all, we thank the families who have and will participate in the study. This study is funded by the Thrasher Research Fund.

Contributors GJ-S, BAR, JK and SML designed the randomised clinical trial. SML, GJ-S, BAR, TRH, LMC, JK, DM, AJW and EM-0 developed the study protocol. GJ-S is the principal investigator and protocol chair and TRH is the immunology principal investigator. JK is the protocol co-chair and country principal investigator. EM-0 is the Pediatric Clinical TB lead. GJ-S, BAR and SML are responsible for the statistical design of the trial and data analysis of the primary outcomes. SML is the project director and drafted the statistical analysis plan overseen by BAR, the study biostatistician. SML, DM, AJW, JK and JNE participated in trial implementation and manuscript preparation. TRH designed the immunological studies and oversees the immunological work related to the trial. SML wrote the first draft of the manuscript. All authors critically revised, read and approved the final manuscript.

Funding This work was supported by the Thrasher Research Fund, National Institute of Allergy and Infectious Diseases (NIAID), Fulbright programme awarded to the Northern Pacific Global Health Fellows Program by the Fogarty International Center of the National Institutes of Health (NIH/Fogarty), and National Center for Advancing Translational Sciences at National Institutes of Health (NIH) (Thrasher to GJ-S, NIH/NIAID K23Al120793 to SML, NIH/NIAID 2K24Al137310 to TRH, NIH/ Fogarty R25TW009345 to AJW and NIH UL1TR000423 for REDCap).

Competing interests None declared.

Patient consent for publication Not required.

Ethics approval The protocol is approved by the University of Nairobi/Kenyatta National Hospital Ethics and Research Committee (P571/08/2015), Jaramogi Oginga Odinga Teaching and Referral Hospital, University of Washington Institutional Review Board (STUDY00000761), and Kenya Pharmacy and Poisons Board.

Provenance and peer review Not commissioned; externally peer reviewed.

Open access This is an open access article distributed in accordance with the Creative Commons Attribution Non Commercial (CC BY-NC 4.0) license, which permits others to distribute, remix, adapt, build upon this work non-commercially, and license their derivative works on different terms, provided the original work is properly cited, appropriate credit is given, any changes made indicated, and the use is non-commercial. See: http://creativecommons.org/licenses/by-nc/4.0/.

\section{ORCID iDs}

Sylvia M LaCourse http://orcid.org/0000-0002-9809-5997

Grace C John-Stewart http://orcid.org/0000-0002-4301-1573 


\section{REFERENCES}

1 Cotton MF, Schaaf HS, Lottering G, et al. Tuberculosis exposure in HIV-exposed infants in a high-prevalence setting. Int J Tuberc Lung Dis 2008;12:225-7.

2 Madhi SA, Nachman S, Violari A, et al. Primary isoniazid prophylaxis against tuberculosis in HIV-exposed children. $N$ Engl J Med 2011;365:21-31.

3 Martinez L, Lo NC, Cords O, et al. Paediatric tuberculosis transmission outside the household: challenging historical paradigms to inform future public health strategies. Lancet Respir Med 2019;7:544-52.

4 Marais BJ, Gie RP, Schaaf HS, et al. The natural history of childhood intra-thoracic tuberculosis: a critical review of literature from the prechemotherapy era. Int J Tuberc Lung Dis 2004;8:392-402.

5 Marais BJ, Gie RP, Schaaf HS, et al. The clinical epidemiology of childhood pulmonary tuberculosis: a critical review of literature from the pre-chemotherapy era. Int J Tuberc Lung Dis 2004;8:278-85.

6 Gedde-Dahl T. Tuberculous infection in the light of tuberculin matriculation. Am J Hyg 1952;56:139-214.

7 Moyo S, Verver S, Mahomed H, et al. Age-related tuberculosis incidence and severity in children under 5 years of age in Cape Town, South Africa. Int J Tuberc Lung Dis 2010;14:149-54.

8 Comstock GW, Livesay VT, Woolpert SF. The prognosis of a positive tuberculin reaction in childhood and adolescence. Am J Epidemiol 1974;99:131-8.

9 Starke JR. Childhood tuberculosis during the 1990s. Pediatr Rev 1992;13:343-53.

10 Donald PR, Maher D, Qazi S. A research agenda to promote the management of childhood tuberculosis within national tuberculosis programmes. Int J Tuberc Lung Dis 2007;11:370-80.

11 World Health Organization. Latent TB infection: updated and consolidated guidelines for programmatic management, 2018. Available: http://www.who.int/tb/publications/2018/latenttuberculosis-infection/en/

12 Zar HJ, Cotton MF, Strauss S, et al. Effect of isoniazid prophylaxis on mortality and incidence of tuberculosis in children with HIV: randomised controlled trial. BMJ 2007;334:136.

13 Gray DM, Workman LJ, Lombard CJ, et al. Isoniazid preventive therapy in HIV-infected children on antiretroviral therapy: a pilot study. Int J Tuberc Lung Dis 2014;18:322-7.

14 Zunza M, Gray DM, Young T, et al. Isoniazid for preventing tuberculosis in HIV-infected children. Cochrane Database Syst Rev 2017;8:CD006418.

15 Cranmer LM, Kanyugo M, Jonnalagadda SR, et al. High prevalence of tuberculosis infection in HIV-1 exposed Kenyan infants. Pediatr Infect Dis J 2014;33:401-6.

16 Martinez L, le Roux DM, Barnett W, et al. Tuberculin skin test conversion and primary progressive tuberculosis disease in the first 5 years of life: a birth cohort study from Cape Town, South Africa. Lancet Child Adolesc Health 2018;2:46-55.

17 Marquez C, Chamie G, Achan J, et al. Tuberculosis infection in early childhood and the association with HIV-exposure in HIV-uninfected children in rural Uganda. Pediatr Infect Dis J 2016;35:524-9.

18 Bekker A, Du Preez K, Schaaf HS, et al. High tuberculosis exposure among neonates in a high tuberculosis and human immunodeficiency virus burden setting. Int J Tuberc Lung Dis 2012;16:1040-6.

19 Pai M, Denkinger CM, Kik SV, et al. Gamma interferon release assays for detection of Mycobacterium tuberculosis infection. Clin Microbiol Rev 2014;27:3-20.

20 American Academy of Pediatrics. Tuberculosis. In: Kimberlin DW, Brady MT, Jackson MA, eds. Red book 2018-2021 report of the Committee on Infectious Diseases; American Academy of Pediatrics. 31st Edition ed. Elk Grove Village, IL: American Academy of Pediatrics, 2018: 879-853.

21 Lewinsohn DM, Leonard MK, LoBue PA, et al. Official American Thoracic Society/Infectious Diseases Society of America/Centers for Disease Control and Prevention clinical practice guidelines: diagnosis of tuberculosis in adults and children. Clin Infect Dis 2017;64:111-5.

22 Pai M, Behr MA, Dowdy D, et al. Tuberculosis. Nat Rev Dis Primers 2016;2:16076.

23 Andrews JR, Nemes E, Tameris M, et al. Serial quantiferon testing and tuberculosis disease risk among young children: an observational cohort study. Lancet Respir Med 2017;5:282-90.

24 Velasco-Arnaiz E, Soriano-Arandes A, Latorre I, et al. Performance of tuberculin skin tests and interferon- $\gamma$ release assays in children younger than 5 years. Pediatr Infect Dis J 2018;37:1235-41.

25 Mandalakas AM, van Wyk S, Kirchner HL, et al. Detecting tuberculosis infection in HIV-infected children: a study of diagnostic accuracy, confounding and interaction. Pediatr Infect Dis $J$ 2013;32:e111-8.
26 Verhagen LM, Maes M, Villalba JA, et al. Agreement between QuantiFERON $\AA$-TB gold in-tube and the tuberculin skin test and predictors of positive test results in Warao Amerindian pediatric tuberculosis contacts. BMC Infect Dis 2014;14:383.

27 Debulpaep S, Corbière V, Levy J, et al. Contribution of QuantiFERON-TB Gold-in-Tube to the diagnosis of Mycobacterium tuberculosis infection in young children in a low TB prevalence country. Front Pediatr 2019;7:291.

28 Elliot C, Marais B, Williams P, et al. Tuberculin skin test versus interferon-gamma release assay in refugee children: a retrospective cohort study. J Paediatr Child Health 2018;54:834-9.

29 Cranmer LM, Draper HR, Mandalakas AM, et al. High incidence of tuberculosis infection in HIV-exposed children exiting an isoniazid preventive therapy trial. Pediatr Infect Dis J 2018;37:e254-6.

30 Kenya Ministry of Health. National guidelines on management of tuberculosis in children. 3rd edn, 2017. https://www.chskenya.org/ wp-content/uploads/2018/03/National-Guidelines-on-Managementof-Tuberculosis-in-Children.pdf

31 Samandari T, Agizew TB, Nyirenda S, et al. 6-Month versus 36-month isoniazid preventive treatment for tuberculosis in adults with HIV infection in Botswana: a randomised, double-blind, placebo-controlled trial. Lancet 2011;377:1588-98.

32 Rangaka MX, Wilkinson RJ, Boulle A, et al. Isoniazid plus antiretroviral therapy to prevent tuberculosis: a randomised doubleblind, placebo-controlled trial. Lancet 2014;384:682-90.

33 Danel C, Moh R, Gabillard D, et al. A trial of early antiretrovirals and isoniazid preventive therapy in Africa. N Engl J Med 2015;373:808-22.

34 Badje A, Moh R, Gabillard D, et al. Effect of isoniazid preventive therapy on risk of death in West African, HIV-infected adults with high CD4 cell counts: long-term follow-up of the Temprano ANRS 12136 trial. Lancet Glob Health 2017;5:e1080-9.

35 World Health Organization. Global tuberculosis report 2018, 2018. Available: http://apps.who.int/iris/bitstream/handle/10665/274453/ 9789241565646-eng.pdf?ua=1

36 Kenya Ministry of Health. Kenya tuberculosis survey, 2017. Available: https://www.nltp.co.ke/survey-reports-2/

37 Kenyan Ministry of Health, National AIDS and STI Control Program (NASCOP). Guidelines on use of antiretroviral drugs for treating and preventing HIV infection: a rapid advice, 2014. Available: http://healthservices.uonbi.ac.ke/sites/default/files/centraladmin/ healthservices/Rapid\%20Advice\%20Booklet\%202014\%2024\% 20June\%2012\%20noon_0.pdf

38 World Health Organization. Guidelines for intensified tuberculosis case-finding and isoniazid preventive therapy for people living with HIV in resource-constrained settings, 2011. Available: http:// whqlibdoc.who.int/publications/2011/9789241500708_eng.pdf

39 World Health Organization. Guidance for national tuberculosis programmes on the management of tuberculosis in children. 2nd edn, 2014. http://www.who.int/tb/publications/childtb_guidelines/en/

40 Cobelens F, Van Deutekom H, Draayer-Jansen I, et al. Tuberculin skin test reactions by time of reading among Dutch travellers. Int $J$ Tuberc Lung Dis 2003;7:758-63.

41 Tuberculin reaction size on five consecutive days. Bull World Health Organ 1955;12:189-96.

42 Nackers F, Huerga H, Espié E, et al. Adherence to self-administered tuberculosis treatment in a high HIV-prevalence setting: a crosssectional survey in Homa Bay, Kenya. PLoS One 2012;7:e32140.

43 Meissner PE, Musoke P, Okwera A, et al. The value of urine testing for verifying adherence to anti-tuberculosis chemotherapy in children and adults in Uganda. Int $J$ Tuberc Lung Dis 2002;6:903-8.

44 Gerona R, Wen A, Chin AT, et al. Quantifying isoniazid levels in small hair samples: a novel method for assessing adherence during the treatment of latent and active tuberculosis. PLOS One 2016;11:e0155887.

45 Mave V, Chandanwale A, Kinikar A, et al. Isoniazid hair concentrations in children with tuberculosis: a proof of concept study. Int J Tuberc Lung Dis 2016;20:844-7.

46 Division of AIDS National Institute of Allergy and Infectious Diseases. Division of AIDS (DAIDS) table for grading the severity of adult and pediatric adverse events, 2014. Available: http://rsc.tech-res.com/ docs/default-source/safety/daids_ae_grading_table_v2_nov2014.pdf

47 IMPAACT P1041 trial protocol, 2007. Available: https:// impaactnetwork.org/studies/P1041.asp

48 Harris PA, Taylor R, Thielke R, et al. Research electronic data capture (REDCap) - a metadata-driven methodology and workflow process for providing translational research informatics support. J Biomed Inform 2009;42:377-81.

49 Qiagen. QuantiFERON®-TB gold plus (QFT®-Plus) ELISA package insert, 2014. Available: http://www.quantiferon.com/irm/content/PI/ QFT/PLUS/2PK-Elisa/UK.pdf 
50 Rozot V, Patrizia A, Vigano S, et al. Combined use of Mycobacterium tuberculosis-specific CD4 and CD8 T-cell responses is a powerful diagnostic tool of active tuberculosis. Clin Infect Dis 2015;60:432-7.

51 Armand M, Chhor V, de Lauzanne A, et al. Cytokine responses to quantiferon peptides in pediatric tuberculosis: a pilot study. $\mathrm{J}$ Infect 2014;68:62-70.

52 Kim SY, Park MS, Kim YS, et al. The responses of multiple cytokines following incubation of whole blood from TB patients, latently infected individuals and controls with the TB antigens ESAT-6, CFP10 and TB7.7. Scand J Immunol 2012;76:580-6.

53 Lighter-Fisher J, Peng C-H, Tse DB. Cytokine responses to QuantiFERON $\otimes$ peptides, purified protein derivative and recombinant ESAT-6 in children with tuberculosis. Int J Tuberc Lung Dis 2010;14:1548-55.

54 Rubbo P-A, Nagot N, Le Moing V, et al. Multicytokine detection improves latent tuberculosis diagnosis in health care workers. J Clin Microbiol 2012;50:1711-7.

55 Biselli R, Mariotti S, Sargentini V, et al. Detection of interleukin-2 in addition to interferon-gamma discriminates active tuberculosis patients, latently infected individuals, and controls. Clin Microbiol Infect 2010;16:1282-4.

56 Warr AJ, Shah JA, LaCourse SM, et al. Mycobacterium tuberculosis antigen-specific T-cell responses in HIV-infected mothers and their infants. In: Keystone symposia on tuberculosis: translating scientific findings for clinical and public health impact. Whistler, British Columbia, Canada, 2018.

57 Comstock GW, Ferebee SH, Hammes LM. A controlled trial of community-wide isoniazid prophylaxis in Alaska. Am Rev Respir Dis 1967:95:935-43.

58 Comstock GW, Baum C, Snider DE. Isoniazid prophylaxis among Alaskan Eskimos: a final report of the bethel isoniazid studies. Am Rev Respir Dis 1979;119:827-30.

59 Smieja MJ, Marchetti CA, Cook DJ, et al. Isoniazid for preventing tuberculosis in non-HIV infected persons. Cochrane Database Syst Rev 2000:CD001363.
60 Akolo C, Adetifa I, Shepperd S, et al. Treatment of latent tuberculosis infection in HIV infected persons. Cochrane Database Syst Rev 2010:CD000171.

61 Ayele HT, Mourik MSMvan, Debray TPA, et al. Isoniazid prophylactic therapy for the prevention of tuberculosis in HIV infected adults: a systematic review and meta-analysis of randomized trials. PLoS One 2015;10:e0142290.

62 Frigati LJ, Kranzer K, Cotton MF, et al. The impact of isoniazid preventive therapy and antiretroviral therapy on tuberculosis in children infected with HIV in a high tuberculosis incidence setting. Thorax 2011;66:496-501.

63 Farhat M, Greenaway C, Pai M, et al. False-positive tuberculin skin tests: what is the absolute effect of BCG and non-tuberculous mycobacteria? Int J Tuberc Lung Dis 2006;10:1192-204.

64 Mancuso JD, Mody RM, Olsen CH, et al. The long-term effect of Bacille Calmette-Guérin vaccination on tuberculin skin testing. Chest 2017;152:282-94.

65 Lifschitz M. The value of the tuberculin skin test as a screening test for tuberculosis among BCG-vaccinated children. Pediatrics 1965;36:624-7.

66 Kaguthi G, Nduba V, Murithi W, et al. The incidence of nontuberculous mycobacteria in infants in Kenya. $J$ Trop Med 2019;2019:1273235

67 LaCourse SM, Wagner AD, Cranmer LM, et al. Brief report: high programmatic isoniazid preventive therapy (IPT) use in pregnancy among HIV-infected women. J Acquir Immune Defic Syndr 2019;82:41-5.

68 Garessus EDG, Mielke H, Gundert-Remy U. Exposure of infants to isoniazid via breast milk after maternal drug intake of recommended doses is clinically insignificant irrespective of metaboliser status. A physiologically-based pharmacokinetic (PBPK) modelling approach to estimate drug exposure of infants via breast-feeding. Front Pharmacol 2019;10:5 